\title{
THE WORD PROBLEM FOR GEOMETRICALLY \\ FINITE GROUPS
}

This note is based on J. W. Cannon's paper [2], in which he gives, among other things, a solution of the word problem for cocompact groups of isometries of hyperbolic space of dimension $d \geqslant 1$. We have thought it worthwhile to show how Cannon's ideas can be rearranged to give a rather simple solution of this problem. Although the result obtained here is in important respects weaker than that of Cannon, it is in other respects more general. In particular, it also works for cocompact groups of isometries of Euclidean space and discrete groups of isometries of hyperbolic space whose quotients have finite volume. If one accepts as part of the initial information the Nielsen region for $G$, then it can also be used to give a solution of the word problem for geometrically finite groups of isometries of hyperbolic 3-space.

We view our result as an instance of the Todd-Coxeter coset enumeration algorithm (see [5] for a good description), which we formulate as follows. Let a group $G$ have a presentation $\langle X: R\rangle$, with $G=F / N$, where $F$ is a free group with basis $X$ and $N$ is the normal closure of the subset $R$ in $F$. For any natural number $n$, let $R(n)$ be the set of all words in $X$ of the form $w=u r u^{-1}$ for $r$ in $R$ and $u$ an element of $F$ represented by a reduced word of length $|u| \leqslant n$. Let $F(n)$ be the set of all initial segments of words $w$ in $R(n)$. Let $w \sim_{n} w^{\prime}$ be the equivalence relation on $F(n)$ defined by setting $w_{1} w_{2} \sim_{n} w_{1} w_{0} w_{2}$ whenever both are in $F(n)$ and $w_{0}$ is in $R(n)$.

DEFINITION. Let $f$ be any function $f: \mathbb{N} \rightarrow \mathbb{N}$. We say that the presentation $\langle X: R\rangle$ is $f$-decidable if, given any element in $F$ represented by a word $w$ of length $|w|=m$, then $w \in N$ if and only if $w \sim_{n} 1$ for $n=f(m)$.

The Todd-Coxeter algorithm shows that every presentation is $f$-decidable for some function $f$, although, of course, for the general presentation it may not be possible to choose $f$ to be recursive. Indeed, the word problem for $G$ is decidable in the usual sense if and only if $G$ has a presentation that is $f$ decidable for some recursive function $f$. If $G$ is finite, it is possible to choose $f$ as a constant function. If the presentation satisfies Dehn's algorithm (in Cannon's

* W.J.F. was supported in part by NSF Grant 81-02469.

** R.C.L. gratefully acknowledges partial support from the National Science Foundation and from the SERC (U.K.), and the hospitality of the University of Birmingham. 
extended sense), then $f$ can be taken to coincide with the identity function $f(m)=m$ for all $m$ greater than some $M$.

In these terms, our result may be formulated as follows.

THEOREM 1. (i) Let $G$ be a cocompact group of isometries of hyperbolic or Euclidean space of dimension $d \geqslant 2$, and let $\langle X: R\rangle$ be a presentation for $G$ obtained from a Dirichlet region for $G$ in the usual fashion. Then there is a natural number $s$ such that $\langle X: R\rangle$ is $f$-decidable for the quadratic function $f(m)=s m^{2}$.

(ii) Let $G$ be a discrete group of isometries of hyperbolic d-space such that either $d \geqslant 2$ and $H / G$ has finite volume or $d=3$ and $G$ is geometrically finite, and let $\langle X: R\rangle$ be a presentation for $G$ obtained from a Dirichlet region in the usual fashion. Then there is a natural number $s$ such that $\langle X: R\rangle$ is $f$-decidale for the function $f(m)=\left[e^{s m}\right]$, the greatest integer in $e^{s m}$.

Remarks. 1. It does not seem to be known for $H$, except in the case $d=2$, whether the sets $T^{*}(n)$ defined below are simply connected.

2. From the fact that these groups can be represented as finitely generated matrix groups, it follows that they are residually finite and hence have decidable word problem. But this argument does not yield any geometric insight, nor any usable algorithm.

\section{THE MAIN LEMMA}

In what follows $U$ denotes either Euclidean $d$-space $E$ or hyperbolic $d$-space $H$ of dimension $d \geqslant 2$, and $G$ is a discrete group of isometries of $U$ such that (A) $U=$ $H$ or $U=E, d \geqslant 2$, and $U / G$ is compact, (B) $U=H, d \geqslant 2$, and $U / G$ has finite volume, or (C) $U=H, d=3$, and $G$ is geometrically finite but not a Fuchsian group. We take as our model for $H$ the interior of the closed unit ball $D^{d}$ in $\mathbb{R}^{d}$, with the Poincare metric $\mathrm{ds} s^{2}=4 \mathrm{~d} x^{2} /\left(1-r^{2}\right)^{2}$, where $\mathrm{d} x^{2}=\mathrm{d} x_{1}^{2}+\cdots+\mathrm{d} x_{d}^{2}$ is the standard Euclidean metric on $\mathbb{R}^{d}$. We can choose a base point $b$ in $U$ that is fixed by no non-trivial element of $G$ and define a (closed) Dirichlet region $\Delta=$ ( $p \in U$ : for all $g \in G, d(p, b) \leqslant d(p, g b)$ ), where $d(p, q)$ is the distance between $p$ and $q$. If we are in Case $C$ we choose $b$ so that it is in the interior of the Nielsen region for $G$ (see [1] or [4] for definitions and details of $N$ ). Then $\Delta$ is a convex cell bounded by a finite number of $(d-1)$-dimensional cells. The set of all images $g \Delta$ of $\Delta$ for $g$ in $G$ then provides a tessellation $T$ of $U$, that is, a cell decomposition with the $g \Delta$ as $d$-cells. Note that all of the cells of $T$ are convex. In each case we shall construct from $T$ a dual $G$-invariant complex $T^{*}$ whose 2-skeleton $K$ serves as a Cayley complex for the presentation of $G$. 
First we consider Case A, that $U / G$ is compact. Let $T^{\prime}$ be a $G$-equivariant barycentric subdivision of $T$ with the points $g b$ chosen as barycenters of the $g \Delta$ and with the edges geodesic arcs, and let $T^{\bullet}$ be the tessellation of $U$ dual to $T$ with respect to the barycentric subdivision $T^{\prime}$. The vertices of $T^{\bullet}$ are the $g b$ for all $g$ in $G$, and the $d$-cells of $T^{*}$ are the stars in $T^{\prime}$ of the vertices of $T$. We note that the cells of $T^{\bullet}$ are intersections of stars of vertices in $T^{\prime}$ and need not be convex. A 1-cell, or edge, of $T^{\circ}$ will be made up of two edges from $T^{\prime}$, lying in two adjacent $g_{1} \Delta, g_{2} \Delta$ that have a face in common. A 2-cell of $T^{\bullet}$ will be the union of 2-cells in $T^{\prime}$, lying in a set of $g \Delta$ that form a cycle about a $(d-2)$-cell of $T$. In Case A we define $T^{*}=T^{\bullet}$.

We need to modify the construction of $T^{\bullet}$ and $T^{*}$ if $U / G$ is not compact. Let $P$ be the union of a disjoint, $G$-invariant family of closed horoballs around the parabolic fixed points of $G$ such that $b$ is not in $P$. Let $H^{\prime}=H-\operatorname{int}(P)$, and let $\Delta^{\prime}=\Delta \cap H^{\prime}$.

Next we consider Case B, where $U=H$ and $U / G$ has finite volume. Let $T^{\circ}$ be the tessellation of $H^{\prime}$ whose cells are components of intersections of cells of $T$ with $H^{\prime}$ or $\partial H^{\prime}$. Let $T^{\prime}$ be a $G$-equivariant barycentric subdivision of $T^{\circ}$ with the points $g b$ as barycenters of the $g \Delta^{\prime}$ and the edges geodesic arcs unless they are in $\partial H^{\prime}$, and let $T^{\bullet}$ be the tessellation of $H^{\prime}$ dual to $T^{\circ}$ with respect to $T^{\prime}$. Let $T^{*}$ be the subcomplex of $T^{\bullet}$ of cells which are disjoint from $\partial H^{\prime}$.

We need a further modification in Case $\mathrm{C}$, where $U=H$ and $G$ is geometrically finite. Let $N$ be the Nielsen region for $G$ and let $N^{\prime}=N \cap H^{\prime}$. Then $G$ acts on $N^{\prime}$ with fundamental region $\Delta \cap N^{\prime}=\Delta^{\prime} \cap N$. Let $T^{\circ}$ be the tessellation of $N^{\prime}$ whose cells are components of intersections of cells of $T$ with $N^{\prime}$ or $\partial N \cap H^{\prime}$ or $N \cap \partial H^{\prime}$. Let $T^{\prime}$ be a $G$-equivariant barycentric subdivision of $T^{\circ}$ with the $g b$ as barycenters of the $g\left(\Delta \cap N^{\prime}\right)$ and the edges geodesic arcs unless they are in $\partial N^{\prime}$, and let $T^{\bullet}$ be the tessellation of $N^{\prime}$ dual to $T^{\circ}$ with respect to $T^{\prime}$. Let $T^{*}$ be the subcomplex of $T^{\bullet}$ of cells which are disjoint from $\partial N^{\prime}$. We shall often abuse notation by writing $T^{\bullet}$ instead of $\left|T^{\bullet}\right|$ and $T^{*}$ instead of $\left|T^{*}\right|$.

LEMMA 1. If $r \in \mathbb{R}$, then $B(r) \cap T^{\bullet}$ is simply connected, where $B(r)$ is the ball in $U$ with radius $r$ and center $b$. In addition, $T^{*}$ and $T^{*}$ are simply connected.

Proof. We divide the proof into three cases, depending on the type of $G$.

(A) In this case $T^{*}=T^{*}=U$ and the lemma is clear.

(B) Here $T^{\bullet}=H^{\prime}$. For each $r \in \mathbb{R}$ there is a retraction of $B(r)$ onto $B(r) \cap H^{\prime}$, so $B(r) \cap T^{\bullet}$ is simply connected. This implies that $T^{\bullet}$ is simply connected. Since $T^{\bullet}$ collapses to $T^{*}, T^{*}$ is simply connected.

(C) In this case $T^{\bullet}=N^{\prime}=N \cap H^{\prime}$. If $r \in \mathbb{R}$ then $B(r) \cap N$ is simply connected since it is convex, and $B(r) \cap T^{*}$ is simply connected since there is a retraction of 
$B(r) \cap N$ onto $B(r) \cap T^{\bullet}$. This implies that $T^{\bullet}$ is simply connected, and $T^{*}$ is simply connected since $T^{\bullet}$ collapses to $T^{*}$.

The 2-skeleton, $K$, of $T^{*}$ can be made into a Cayley complex as follows. If $X$ is the set of elements $x$ in $G$ such that $\Delta$ and $x \Delta$ have exactly a $(d-1)$-dimensional face in common, then 1 is not in $X, x \in X$ implies $x^{-1} \in X$, and $X$ generates $G$. At each vertex $g b$ of $K$ there is exactly one directed edge $e$ of $K$ from $g b$ to $\bar{x} g b$ for each $x$ in $X$, where $\bar{x}$ is the image of $x$ in $G$, and we assign to $e$ the label (or color) $\Phi(e)=x$. Then every path $\gamma=e_{1} \cdots e_{n}$ in $K$ acquires as label a word $\Phi(\gamma)=$ $\Phi\left(e_{1}\right) \cdots \Phi\left(e_{n}\right)$ over $X$. Conversely, given a word $w$ over $X$ and a vertex $g b$ of $K$, there is a unique path $\gamma$ in $K$ beginning at $g b$ and with label $\Phi(\gamma)=w$; moreover, $\gamma$ will end at $\bar{w} g b$. In particular, $\gamma$ will be a closed path just in case $w$ represents the trivial element 1 in $G$.

For every $n \in \mathbb{N}$ let $T^{*}(n)$ be the union of all $d$-cells of $T^{*}$ that contain a vertex $\bar{w} b$, where $w$ is a word of length $|w| \leqslant n$, and let $K(n)=T^{*}(n) \cap K$, the 2skeleton of $T^{*}(n)$. Let $T^{*}(n)$ be the union of $T^{*}(n)$ with all $d$-cells of $T^{*}-\operatorname{int}\left(T^{*}\right)$ which intersect $T^{*}(n)$ in a $(d-1)$-dimensional face. Note that $T^{*}(n)$ collapses to $T^{*}(n)$.

If $\gamma$ is a closed path in $K$, then $\gamma$ bounds a disc (not necessarily embedded) in $T^{*}$ since $T^{*}$ is simply connected. Since the cells of $T^{*}$ are balls, the disc can be pushed, keeping all boundary points fixed, into the 2-skeleton $K$ of $T^{*}$. Thus $K$ is simply connected, and a closed path in $K(n)$ is contractible in $T^{*}(n)$ just in case it is contractible in $K(n)$. Furthermore, $G$ has a (symmetrized) presentation $\langle X: R\rangle$ where $R$ is the set of labels on the closed paths at $b$ in $K$ that bound the 2-cells of $K$ incident with $b$ (that is, $G$ has an ordinary presentation with generators $X$ and relators $R$ together with all relators $x x^{-1}$ for $x \in X$ ).

Let $w$ be a word in $X$ which represents the trivial element 1 in $G$. Then its associated path $\gamma$ bounds a disc in $K$. We want to show that $\gamma$ bounds a disc in $K$ whose 'combinatorial area' is bounded by a function of $|w|$. The following approach towards 'combinatorial area' seems convenient. Let $K^{1}$ be the 1skeleton of $K$. Let $f: D^{2} \rightarrow K$ be a (possibly singular) disc in $K$, with $f\left(\partial D^{2}\right) \subset$ $K^{1}, f$ transverse to $K^{1}$, and $f$ piecewise smooth. We define its complexity $c(f)$ to be 1 plus the sum, over all components $\sigma$ of $f^{-1}\left(K-K^{1}\right)$, of $\left|\operatorname{degree}\left(\left.f\right|_{\bar{\sigma}}\right)\right|$. Here degree $\left(\left.f\right|_{\bar{\sigma}}\right)$ is the topological degree of $\left.f\right|_{\bar{\sigma}}:(\bar{\sigma}, \partial \bar{\sigma}) \rightarrow(f(\bar{\sigma})$, $\partial f(\bar{\sigma}))$. There is a homotopy of $f$ to a disc which is cellular with respect to some cell structure on $D^{2}$, and $c(f)$ gives an upper bound on the number of 2-cells needed for this.

Theorem 1 is based on the following lemma.

LEMMA 2. (i) Given $U$ and $G$ in Case $A$ and $\langle X: R\rangle$ as above, there exist positive integers $s$ and $t$ with the following property. If $w$ is any word over $X$ of 
length $|w|=m$, then $w$ represents the trivial element 1 in $G$ if and only if the path $\gamma$ at $b$ in $K$ with label $\Phi(\gamma)=w$ is closed and bounds a piecewise smooth disc $f$ : $D^{2} \rightarrow K(s m)$ with complexity $c(f) \leqslant t m^{2}$.

(ii) Given $U$ and $G$ in Cases $B$ or $C$ and $\langle X: R\rangle$ as above, there exist positive integers $r, s$, and $t$ with the following property. If $w$ is any word over $X$ of length $|w|=m$, then $w$ represents the trivial element 1 in $G$ if and only if the path $\gamma$ at $b$ in $K$ with label $\Phi(\gamma)=w$ is closed and bounds a piecewise smooth disc $f: D^{2} \rightarrow K\left(\left[r \mathrm{e}^{s m}\right]\right)$ with complexity $c(f) \leqslant t m \mathrm{e}^{s m}$.

Now assume the lemma, and let $h$ be the maximal length of a relator in $R$. Let $w$ be a word over $X$ of length $|w|=m$, and let $\gamma$ be the path at $b$ in $K$ with label $\Phi(\gamma)=w$. If $\gamma$ is closed and bounds a piecewise smooth disc $f$, then $\gamma$ bounds a cellular disc with at most $c(f)$ 2-cells. There is a homotopy of $\gamma$ to 1 by successively pushing across the 2-cells. The number of edges in $K^{1}$ of the successive boundary curves is bounded by $m+(h-2) c(f)$ since the number goes up at most by $h-2$ for each 2-cell, so $w \sim_{n} 1$ for $n=m+(h-2) c(f)$. The theorem now follows directly from the lemma, which implies that the presentation is $g$-decidable for $g(m)=m+(h-2) t m^{2}$ in Case A and for $g(m)=m+(h-2) m \mathrm{e}^{s m}$ in Cases B and C.

\section{Proof of Lemma 2}

Lemma 2 will be derived from the following two lemmas.

LEMMA 3. There exist positive integers $s_{1}$ and $t_{1}$ such that, for all positive integers $n, K(n) \subseteq B\left(s_{1} n+t_{1}\right)$.

LEMMA 4. Let $k(m)=m$ in Case $A$ and $k(m)=e^{m / 2}$ in Cases $B$ and C. Then there are positive integers $s_{2}$ and $t_{2}$ such that for all positive integers $m$, $\left(T^{\bullet} \cap B(m)\right) \subseteq T^{\bullet}\left(\left[s_{2} k(m)+t_{2}\right]\right)$.

Proof of Lemma 3. Since $X$ is finite we may choose an integer $s_{1}$ such that $s_{1} \geqslant d(b, x b)$ for all $x \in X$. If $p$ and $q$ are vertices of $K$ which are joined by an edge in $K$, then $d(p, q) \leqslant s_{1}$. Thus if $g \in G$ is given by a word $w$ of length $|w|=n$, then $d(b, g b) \leqslant s_{1} n$. If $p$ is any point of $K(n)$, then $p$ lies on a 2-cell $\sigma$ of $K$ containing some vertex $g b$ for $g$ given by a word $w$ of length $|w| \leqslant n$. Each 2-cell containing $b$ has finite diameter, and there are only finitely many 2 -cells. Let $t_{1}$ be any integer not less than the diameters of the 2-cells in $K$ containing $b$. Then every 2-cell in $K$ has diameter at most $t_{1}$. Since $p$ and $g b$ lie on the same 2-cell $\sigma$, $d(b, p) \leqslant d(b, g b)+d(g b, p) \leqslant s_{1} n+t_{1}$ and hence $K(n) \subseteq B\left(s_{1} n+t_{1}\right)$.

Proof of Lemma 4. Let $\mu$ be any positive real number. (The choice of $\mu$ affects both the efficiency of the algorithm and the amount of work involved in computing $t_{2}$.) Since $G$ acts properly discontinuously on $T^{\bullet}$ and $\Delta^{\prime \prime}=\Delta \cap T^{\bullet}$ is 
compact, the number of elements $g$ in $G$ with $d\left(\Delta^{\prime \prime}, g \Delta^{\prime \prime}\right) \leqslant \mu$ is finite. Let $t_{2}=\max \left(|g|: d\left(\Delta^{\prime \prime}, g \Delta^{\prime \prime}\right) \leqslant \mu\right)$. Let $p$ be any point of $T^{\circ}$ with $0<d(p, b) \leqslant m$. Then there is an $\operatorname{arc} \lambda$ in $T^{\bullet}$ from $b$ to $p$ of length $L \leqslant k(m)$. [This uses [3] in Cases B and C. ] Let $r=[L / \mu]+1$, whence $(r-1) \mu \leqslant L \leqslant r \mu$. Subdivide $\lambda$ into segments by points $b=p_{0}, p_{1}, \ldots, p_{r}=p$ such that each $d\left(p_{i-1}, p_{i}\right) \leqslant \mu$. Each $p_{i}$ is contained in $g_{i} \Delta^{\prime \prime}$ for some $g_{i}$ in $G$, where $g_{0}=1$. Since $d\left(\Delta^{\prime \prime}, g_{i-1}^{-1} g_{i} \Delta^{\prime \prime}\right)=$ $d\left(g_{i-1} \Delta^{\prime \prime}, g_{i} \Delta^{\prime \prime}\right) \leqslant d\left(p_{i-1}, p_{i}\right) \leqslant \mu$, each $\left|g_{i-1}^{-1} g_{i}\right| \leqslant t_{2}$. Thus

$$
\left|g_{r}\right|=\left|\prod_{i=1}^{r} g_{i-1}^{-1} g_{i}\right| \leqslant \sum_{i=1}^{r}\left|g_{i-1}^{-1} g_{i}\right| \leqslant t_{2} r
$$

Since $r \leqslant(L / \mu)+1$, this gives $\left|g_{r}\right| \leqslant s_{2} k(m)+t_{2}$, where $s_{2}$ is any natural number with $s_{2} \geqslant t_{2} / \mu$. Thus $p$ is in $T^{*}\left(\left[s_{2} k(m)+t_{2}\right]\right)$.

Note that we can choose larger constants $s_{1}^{\prime}$ and $s_{2}^{\prime}$ so that $K(n) \subseteq B\left(s_{1}^{\prime} n\right)$ in Lemma 3 and $\left(T^{\bullet} \cap B(m)\right) \subseteq T^{\bullet}\left(\left[s_{2}^{\prime} k(m)\right]\right)$ in Lemma 4. This makes the final algorithm less efficient, but it makes the statement and notation simpler. We shall use these simpler forms, and shall leave it to the interested reader to make the necessary changes for increased efficiency.

Proof of Lemma 2. $\Leftrightarrow$ ) This direction is clear, since if $\gamma$ is closed then $w$ represents 1 in $G$.

$\Leftrightarrow$ Let $w$ be a word over $X$ of length $|w|=m$ which represents 1 in $G$. The path $\gamma$ at $b$ in $K$ with label $w$ is contained in $K(m)$, and hence, by Lemma 3, in $T^{\bullet} \cap B\left(s_{1}^{\prime} m\right)$. Coning $\gamma$ to $b$ gives a (singular) disc $f: D^{2} \rightarrow B\left(s_{1}^{\prime} m\right)$ whose image has boundary $\gamma$ and is a union of $2 m$ geodesic triangles in $B\left(s_{1}^{\prime} m\right)$. In Cases B and $C$ the disc $f\left(D^{2}\right)$ will not be in $T^{\bullet} \cap B\left(s_{1}^{\prime} m\right)$ if it intersects the horoballs $P$, but by retracting along geodesics asymptotic to the parabolic points we get a new disc, which we still call $f$, whose image $f\left(D^{2}\right)$ lies in $T^{\bullet} \cap B\left(s_{1}^{\prime} m\right)$ and is made up of geodesic pieces and pieces in the horospheres that bound $P$. We can then deform $f$ further to smooth the corners where the geodesic pieces meet the pieces of horospheres. Let $v$ be the maximum length of an edge in $K^{1}$. In Case $A$ the disc $f\left(D^{2}\right)$ is a union of $2 m$ geodesic triangles, each with one edge of length $\leqslant v$ and the other two edges of length $\leqslant s_{1}^{\prime} m$. In Cases $B$ and $C$ the disc $f\left(D^{2}\right)$ is a union of $2 m$ 'smooth triangles', each with one boundary arc of length $\leqslant v$ and two boundary arcs of length $\leqslant \mathrm{e}^{s_{1}^{\prime} m / 2}$.

Let $k(m)=m$ in Case A and $k(m)=\mathrm{e}^{m / 2}$ in Cases B and C. By Lemma 4, $f\left(D^{2}\right)$ lies in $T^{\circ}\left(s_{2}^{\prime} k\left(s_{1}^{\prime} m\right)\right)$. As in the proof of Lemma 4, one can show that there is a positive integer $j$ so that $f\left(D^{2}\right)$ intersects at most $j m k\left(s_{1}^{\prime} m\right) d$-cells of $T^{*}$. Since $T^{*}$ collapses to $T^{*}$ and all of the cells of $T^{*}$ are balls, $f$ can be deformed, keeping its boundary fixed, into a piecewise smooth disc $g: D^{2} \rightarrow K$. By a subdivision argument like the one in Lemma 4 , we can show that 
$c(g) \leqslant J m k\left(s_{1}^{\prime} m\right)$ for some positive integer $J$. Lemma 2 follows with $s=s_{1}^{\prime}$ and $t=J s_{1}^{\prime}$ in Case A and $r=s_{2}^{\prime}, s=\left[1+s_{1}^{\prime} / 2\right]$ and $t=J$ in Cases B and C.

\section{REFERENCES}

1. Beardon, A. F., The Geometry of Discrete Groups, Springer-Verlag, Berlin, Heidelberg, New York, 1983.

2. James W. Cannon, 'The Combinatorial Structure of Cocompact Discrete Hyperbolic Groups', Geom. Dedicata 16 (1984), 123-148.

3. William J. Floyd, 'Group Completions and Limit Sets of Kleinian Groups', Invent. Math. 57 (1980), 205-218.

4. William J. Harvey (ed.), Discrete Groups and Automorphic Functions, Academic Press, New York, 1977.

5. Johnson, D. L., Topics in the Theory of Group Presentations, Cambridge Univ. Press, Cambridge, 1980.

Authors' addresses:

W. J. Floyd,

Department of Mathematics, VPI \& SU,

Blacksburg,

VA 24061,

U.S.A.

A. H. M. Hoare,

Department of Mathematics,

The University of Birmingham, P.O. Box 363,

Birmingham B15 2TT.

England

R. C. Lyndon,

Department of Mathematics, University of Michigan,

Ann Arbor,

MI 48109,

U.S.A.

(Received, February 24, 1984; revised version, July 9, 1985) 